included in the analysis. Logistic regression was used to calculate OR $(95 \% \mathrm{CI})$ for the association of chlamydia with menstrual cycle adjusted by demographics and behavioural variables.

Results During the study period, there were 10,017 consultations with positive diagnoses in 417 of those with a valid recorded LNMP. Detection rates were $3.8 \%(233 / 6816)$ in the follicular and $4.8 \%$ (184/3831) in the luteal phase of the menstrual cycle (OR $1.2995 \%$ CI $1.1-1.6, p=0.01)$. Detection was significantly associated with the luteal phase (adjusted odds ratio (aOR) 1.4 (95\% CI 1.1-1.8) when adjusted for age, number of male partners, symptoms, inconsistent use of condoms, site of sample and sexual partners overseas/ from overseas. Among women using hormonal contraception, there was no association with the luteal phase (aOR 1.3, 95\% CI 0.9-1.8, $\mathrm{p}=0.18$; among women not using hormonal contraception, association with the luteal phase was significant (aOR 1.6, (95\% CI 1.1 2.3, $p=0.007$ ). The positive stored samples will undergo analysis to quantify bacterial load and determine if mean load differs across the cycle.

Conclusions Chlamydia detection rates are substantially and significantly higher in the luteal phase of the menstrual cycle. Hormonal and immune changes in the female reproductive tract may contribute to an increased burden of chlamydia infection in this phase, illustrated by the lack of association with the menstrual cycle in women using hormonal contraception.

\section{P5.014 WHAT IS THE OPTIMAL TIME TO RESCREEN STI CLINIC VISITORS WITH A UROGENITAL CHLAMYDIA INFECTION?}

doi:10.1136/sextrans-2013-051184.1060

' $\mathbf{J}$ J van der Helm, 'R H Koekenbier, 'M S van Rooijen, ${ }^{1,2,3 \mathrm{H}} \mathrm{J} \mathrm{C}$ de Vries. ${ }^{1} \mathrm{Health}$ Service Amsterdam, Amsterdam, The Netherlands; '2Academic Medical Center, Amsterdam, The Netherlands; ${ }^{3}$ Centre for Infectious Disease Control, National Institute of Public Health and the Environment, Bilthoven, The Netherlands

Background and Aim STI clinic visitors with a urogenital chlamydia infection $(\mathrm{Ct})$ have a high re-infection rate. Retesting can be an effective strategy to prevent onward transmission and late sequelae. The optimal moment to offer a re-test is unknown.

Methods Between May 2012 and January 2013, all heterosexual visitors of the Amsterdam STI clinic, testing positive for urogenital $\mathrm{Ct}$ were offered retesting after receiving diagnosis, treatment and counselling. Participants were randomly assigned for re-testing after 2, 4 or 6 months. Participants were free to choose between two retest options; receive a home collection kit or an email/SMS invitation to return to the clinic for a self collected retest.

Results In total 1784 individuals were included of whom $47 \%$ were male, $74 \%$ were Dutch and the median age was 23 years (IOR 20-26). 779 (44\%) opted for the home collection kit and 1005 (56\%) for re-visiting the clinic. At this point, 795 are eligible to evaluate retesting; 265 home collection kits were returned (75\%) and 237 individuals returned to the clinic for a retest (54\%). Overall, the participation rate did not differ between the assigned time periods. A test result was available for 266, 126 and 49 individuals in the 2, 4 and 6 month group, respectively. The overall positivity rate at 2,4 and 6 months was respectively $8 \%, 6 \%$ and $12 \%$.

Conclusions Based on these preliminary data we found a high test uptake. Possibly because individuals were able to choose their preferred method of retesting. As the participation rate was not affected by the period of the retest and the positivity rate seemed to be highest after 6 months this might be an optimal time interval to offer a retest to STI clinic visitors. We conclude that retesting is feasible in identifying new $\mathrm{Ct}$ infections.

\section{P5.015 CHLAMYDIA TRACHOMATIS SCREENING AND TREATMENT IN PREGNANT WOMEN IN LIMA, PERU}

doi:10.1136/sextrans-2013-051184.1061

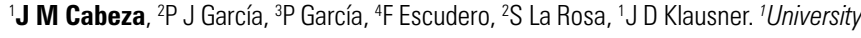
of California in Los Angeles, Los Angeles, CA, United States; ${ }^{2}$ Universidad Peruana Cayetano Heredia, Lima, Peru; 'Instituto Nacional Materno Perinatal, Lima, Peru; ${ }^{4}$ Hospital Nacional Arzobispo Loayza, Lima, Peru

Background Chlamydia trachomatis (CT), the most common bacterial STD and asymptomatic in most women, causes significant adverse outcomes in pregnancy but no programmes routinely conduct prenatal screening in Latin America. To prepare for a clinical trial of CT screening and treatment in pregnancy, we determined the feasibility and acceptability of routine chlamydia screening, patient and partner adherence to treatment, and chlamydia prevalence in pregnant women in Lima, Peru.

Methods We conducted a prospective study of pregnant women $>$ 16 years of age at two large urban maternity hospitals in Lima. We offered chlamydia screening to pregnant women during their first prenatal visit using self-collected vaginal swabs with APTIMA Combo $2^{\circledR}$ Assay (Hologic Gen-Probe, San Diego, CA). CT positive patients were contacted within 14 days of testing and were asked to bring partner(s) for counselling and offered concurrent patient partner treatment (CPPT) with 1 gramme of oral azithromycin. Unaccompanied patients received counselling and treatment in the clinic and expedited partner therapy (EPT) for partners. We performed a test of cure $>3$ weeks after treatment.

Results Over 2 months, we approached 646 women for the study and enrolled 603 (93.5\%). The average (+/- standard deviation) age was $27.2+6.9$ years with an average $2.3+2.5$ lifetime partners and an average gestational age of $26.3+10.5$ weeks. Chlamydia prevalence was $10.0 \%+3.9 \%$. Of 39 CT positive patients contacted so far, $35(90 \%)$ have received treatment. Of those, $46 \%$ received CPPT, $49 \%$ EPT and $5 \%$ had no contactable partners. Treatment and test of cure are ongoing.

Conclusion Chlamydia screening in pregnancy was feasible and highly acceptable in two large urban maternity hospitals in Peru. The prevalence of CT infection was high. Our settings are optimal for a clinical trial of CT screening and treatment to prevent adverse pregnancy outcomes.

\section{P5.016 WITHDRAWN BY AUTHOR}

\section{P5.017 THE AUSTRALIAN CHLAMYDIA CONTROL EFFECTIVENESS PILOT (ACCEPT): EARLY RESULTS FROM A RANDOMISED TRIAL OF ANNUAL CHLAMYDIA SCREENING IN GENERAL PRACTISE}

doi:10.1136/sextrans-2013-051184.1062

'J Hocking, 'M Temple-Smith, ${ }^{2} \mathrm{R}$ Guy, ${ }^{1} \mathrm{~F}$ Kong, ${ }^{3} \mathrm{~N}$ Low, ${ }^{2 B}$ Donovan, ${ }^{2} \mathrm{M}$ Law, ${ }^{2} \mathrm{~J}$ Kaldor, ' $\mathrm{J}$ Gunn, ${ }^{1,4} \mathrm{C}$ Fairley. ${ }^{1}$ University of Melbourne, Carlton, Australia; ${ }^{2}$ Kirby Institute, Sydney, Australia; ${ }^{3}$ University of Bern, Bern, Switzerland; " Melbourne Sexual Health Centre, Carlton, Australia

Background ACCEPt is a cluster randomised controlled trial to evaluate annual opportunistic chlamydia screening for 16-29 year olds in general practise (GP). Towns in which GP clinics are enrolled, are randomised to receive a multifaceted intervention to increase chlamydia testing or continue usual practise. The primary outcome is change in chlamydia prevalence amongst GP patients in each town. We report some early results on testing uptake, a secondary outcome.

Methods From July 2010-December 2011, we enrolled 787 GPs in 150 clinics (response rate $>80 \%$ ) in 54 towns. Chlamydia testing rates (the proportion who consult a GP and have a test during 12 months) and re-testing rates (proportion who are re-tested within 12 ( \pm 3 ) months following a negative or within 3 months following a positive test) were calculated. We compared testing between intervention and control towns from July 2011 to Sept 2012. All analyses are adjusted for intracluster correlation within clinics. 
Result We analysed a total of 21,335 tests in intervention and 13,992 in control clinics. In clinics with 12-15 months intervention period (11 clinics), annual testing has increased by $47 \%$ (from $15.1 \%$ to $22.3 \%$ ) in females, $88 \%$ (from $6.3 \%$ to $11.9 \%$ ) in males and $61 \%$ overall (from $11.6 \%$ to $18.7 \%$ ). After increasing from $7.9 \%$ to $11.8 \%$ in the first 12 months, control clinic testing dropped to $9.7 \%$ in 2012. The relative risk of testing in intervention compared with control clinics is 1.9 (95\% CI: 1.8, 2.0). Of 2876 testing positive, $38 \%$ retest within 3 months and of those testing negative, 22\% retest within 12 months in both trial arms.

Conclusions Nearly twice as many are being tested in the intervention and testing rates are increasing. The high participation rate of clinics in all towns puts the study in a strong position to determine if a pragmatic intervention can reduce chlamydia prevalence.

\section{P5.018 IMPROVED DIAGNOSIS OF NON-NEOPLASTIC CONDITIONS OF CERVIX BY LIQUID BASED CYTOLOGY, CELLBOCK IN COMPARISON WITH CONVENTIONAL PAP SMEAR METHOD}

doi:10.1136/sextrans-2013-051184.1063

N N Manoli, N Manoli, S Kulkarni, A P Chandrashekar. JSS Medical College, Mysore, India

Background The Papanicolaou (Pap) smear is the most successful screening test for carcinoma of cervix, mainly as a result of its simplicity, low cost, low false-negative rate and its early detection of preneoplastic lesions. It is also used to diagnose several infectious agents that manifest with specific cytologic features.

The diagnoses of candida, trichomonas, herpes simplex virus, HPV and bacterial vaginosis can be reliably rendered on Pap smears.

Conventional pap smear (CPS) has its limitations. False negatives in CPS may be related to inadequate sampling, inadequate transfer of the sample onto the glass slide or deficiencies in the microscopic assessment of the slide. To overcome these problems, a new slide preparation method namely the Manual Liquid Based Cytology (MLBC) was introduced, where cells are uniformly dispersed by a membrane, from a suspension of cells in a polymer solution.

MLBC helps in detecting number of infestations like Candida, Leptothrix, HPV with koilocytic atypia and bacterial vaginosis, as observed by many authors and us also.

MLBC, which we are following is inexpensive, cost effective method which we have adapted and are comparing it with CPS for its adequacy and utility. The other advantages, is that the residual specimens can be used for ancillary testing like immunocytochemistry by cell block preparation.

Results Number of cases of bacterial vaginosis diagnosed by CPS were 8, LBC 9 and cell block were zero. trichomonas were 4, by CPS andLBC,2bycellblock., herpeswas 1 byall threemethods, inflammatory (NILM) cases were 22 by CPS, 26 by LBC, 12 by cell block. Inadequate smears were 5 by CPS, NIL by LBC, 25 by cell block

Conclusion CPS and LBC with cell block are useful methods in detection of non neoplastic conditions of cervix.

LBC has increased the diagnostic accuracy by $13 \%$ in our study.

\section{P5.019 STRATEGIES INFLUENCING DID-NOT-ATTEND (DNA) RATES IN SEXUALLY TRANSMITTED INFECTION (STI) CLINICS: A RANDOMISED CONTROLLED TRIAL (RCT) ASSESSING THE EFFECTIVENESS OF A VERBAL WARNING MESSAGE GIVEN AT APPOINTMENT BOOKING}

doi:10.1136/sextrans-2013-051184.1064

D Vijeratnam, P Satchithananthan, J Davies, R Patel, D Rowen, E Foley. Sexual Health Department (GUM), Royal South Hants Hospital, Southampton, UK

Background Missed clinic appointments cause significant wastage of health resources worldwide. The majority of cases who
DNA appointments are young and of lower socio-economic status and, conversely, are most likely to carry STIs. Several interventions aimed at reducing DNA appointments such as reminder SMS texts and letters have been studies internationally. Our UK STI department uses interventions such as monthly displays of DNA appointments, SMS reminders and removal of booking privileges to reduce DNA rates. The objective of this study was to assess whether introducing a verbal missed appointment warning at the time of booking has an effect on subsequent DNA and cancellation rates.

Method 108 patients over 16 years old were recruited in this RCT to show a $25 \%$ difference between the groups. Within the first arm the patients were advised that if they did not cancel their appointments in advance and subsequently DNA'ed then they would be obliged to attend a walk-in service in the future. In the second arm the warning was removed. The DNA rates from these two arms were compared initially to each other and then to other previously studied interventions.

Results Interim analysis of results shows a fall in the DNA rates with those who received a verbal warning message at the time of booking matching SMS text and letter reminders rates seen in published data.

Conclusion With financial constraints affecting STI clinics worldwide, it is essential to consider low-cost methods aimed at reducing DNA rates. Whilst we have shown a warning message to be effective, it is also inexpensive and introduction of such a warning system would be beneficial in resource-limited settings.

\section{P5.020 DRIED BLO0D SAMPLES ON PHILTRE PAPER COULD BE A SOLUTION FOR SCREENING OF HIV AND SYPHILIS IN HARD TO REACH POPULATIONS}

doi:10.1136/sextrans-2013-051184.1065

1J F Bencomo-Gomez, 'G Falero-Diaz, ${ }^{1}$ M A 0 Appolinario, ${ }^{2} \mathrm{~A}$ G P Ferreira, ${ }^{2} \mathrm{E} D$ Silva, ${ }^{1}$ A J Werneck de Castro. IInstituto Vital Brazil - Biomarc, Niterói, Brazil; ${ }^{2} I n s t i t u t o$ Oswaldo Cruz - Bio-Manguinhos, Rio de Janeiro, Brazil

Background The high incidence of HIV and syphilis is a health problem in low-income, isolated and large populations, and one of the most affected sectors is the one of pregnant women. Therefore it is necessary to develop and implement methods and procedures to detect and follow new positive cases for those diseases. The aim of this study was to demonstrate the usefulness of the collection of dried blood on philtre paper for the diagnosis of HIV and syphilis in pre-natal screening programmes in areas of difficult access

Methods Dried blood samples on philtre paper were collected and sent to them by mail without any special condition to the laboratory to be processed. They were studied for the presence of antibodies against HIV or syphilis antigens by ELISA. Reactive samples were confirmed by employing a rapid immunochromatography test to both diseases. Finally, classification between HIV type 1 or 2 was performed by rapid immunoblot test.

Results Of the samples tested for HIV by ELISA, the $0.37 \%$ was positive, so 44 samples showed specific antibodies. They all confirmed when rapid immunochromatography test was used. In addition, $100 \%$ of the samples classified as HIV type 1 by using the rapid immunoblot test. In the case of the samples studied for syphilis by ELISA, the positive ones were the $3.12 \%$, being confirmed by the rapid test all 41 positive samples.

Conclusion It was shown that the use of dried blood collected on philtre paper proved to be a cheap, safe and quick way for the screening of infectious diseases, such as HIV and syphilis, in populations as sensitive as pregnant women. It was also found that, although the test of choice for this type of work is the ELISA, other tests such as rapid tests can be used as a confirmatory test 